\title{
LOCAL EXISTENCE OF THE SOLUTION TO A NONLINEAR INVERSE PROBLEM IN GRAVITATION*
}

\author{
BY \\ M. C. JORGE \\ IIMAS-UNAM, Mexico
}

\begin{abstract}
We consider the problem of modeling the external gravitational field of the earth from measurements of its intensity on the surface. The problem is formulated as a nonlinear oblique boundary value problem for the external gravitational potential. The equation for the potential is approximated for small anomalies and solved by a regular perturbation expansion. The convergence of the series is proved using Schauder estimates, and local existence for small anomalies is established. The technique used to prove the convergence of the series is in the same spirit as the Cauchy-Kowalewsky estimates. This study completes previous work done by Backus who proved uniqueness of the solution.
\end{abstract}

1. Introduction. The problem we consider is the one of recovering the external gravitational field of the earth $\mathbf{g}(\mathbf{x})$ from measurements $F(\mathbf{x})=|\mathbf{g}(\mathbf{x})|$ of its intensity on the surface. To simplify matters we assume a spherical earth of radius one which we will denote by $V$ with boundary $S$ and exterior $S_{\mathrm{e}}$. The problem can now be formulated introducing the gravitational potential $v(\mathbf{x})$ which satisfies $\nabla v=-\mathbf{g}$.

The potential satisfies Laplace's equation in $S_{\mathrm{e}}$ and the magnitude $|\nabla v|$ of $\mathbf{g}$, which is the measured field on the surface, is a given function of position. This is $\nabla v \cdot \nabla v=F$ on $S$. The problem to solve is thus

$$
\begin{array}{cc}
\Delta v=0 & \text { for } \mathbf{x} \text { in } S_{\mathrm{e}}, \quad v \rightarrow 0 \quad \text { as }|\mathbf{x}| \rightarrow \infty, \\
\nabla v \cdot \nabla v=F & \text { for } \mathbf{x} \text { on } S .
\end{array}
$$

It has been shown by Backus [1] that the problem stated in Eqs. (I) has a unique solution for each given $F$. However, a proof of existence is not available to date.

In the following sections we prove existence of solutions using a constructive argument which can be used to compute them numerically. We give sufficient conditions on the magnitude of the anomaly of $F$ to guarantee the desired existence.

\footnotetext{
${ }^{*}$ Received January 13, 1986.
} 
2. Formulation of the problem. We want to prove local existence of the following problem:

$$
\Delta v=0 \quad \text { in } S_{e}, \quad(\nabla v \cdot \nabla v)_{S}=F,
$$

$v \rightarrow 0$ at infinity.

Let $(r, \phi, \sigma)$ be the spherical coordinate system (with origin at the center of the sphere $S$ ) where $r$ is the radial component, $\phi$ the longitude, and $\sigma$ the co-latitude.

We shall assume the gravitational field to be predominantly monopolar. This assumption allows us to take the measured field on the surface $S$ in the form $F(\phi, \sigma)=1+$ $\varepsilon h(\phi, \sigma)$, where the function $h$ is the anomaly and $\varepsilon$ a small positive parameter. Accordingly, we take $v$ in the form $v=1 / r+\varepsilon u$ where the monopole $1 / r$ is responsible for the order 1 contribution in $F$. We thus solve a special case of (I) when $F=1+\varepsilon h$. The equations become, after substitution,

$$
\Delta u=0 \quad \text { in } S_{\mathrm{e}}, \quad 2(\partial u / \partial n)_{S}+\varepsilon(\nabla u \cdot \nabla u)_{S}=h
$$

$u \rightarrow 0$ at infinity.

3. Local existence of the solution to problem (II). To solve the boundary value problem (II), we assume an expansion of the solution in the form

$$
u=\sum_{n=1}^{\infty} \varepsilon^{n-1} u_{n}
$$

Inserting this series into the boundary conditions we have

$$
2 \sum_{n=1}^{\infty} \varepsilon^{n-1}\left(\frac{\partial u_{n}}{\partial n}\right)_{S}+\sum_{n=2}^{\infty} \varepsilon^{n-1} B_{n}=h,
$$

where

$$
B_{n}=\sum_{k=1}^{n-1}\left(\nabla u_{k} \cdot \nabla u_{n-k}\right)_{S}, \quad n \geqslant 2 .
$$

Letting the coefficients of equal powers of $\varepsilon$ be equal, we obtain the following sequence of exterior Neumann problems on the unit sphere:

$$
\begin{gathered}
\Delta u_{n}=0 \quad \text { in } S_{e}, \quad n=1,2, \ldots, \\
O(\varepsilon):\left(\frac{\partial u_{1}}{\partial n}\right)_{S}=\frac{1}{2} h, \\
O\left(\varepsilon^{n}\right):\left(\frac{\partial u_{n}}{\partial n}\right)_{S}=-\frac{1}{2} B_{n}=-\frac{1}{2} \sum_{k=1}^{n-1}\left(\nabla u_{k} \cdot \nabla u_{n-k}\right)_{S}, \quad n \geqslant 2 .
\end{gathered}
$$

We will prove local existence of the solution to problem (II) by proving that for a certain set of boundary data $h$, the series (1) satisfies the following:

(a) converges in an appropriate norm;

(b) is harmonic in $S_{\mathrm{e}}$ and continuous on $S$;

(c) converges to zero at infinity;

(d) satisfies the boundary conditions. 
The appropriate spaces to be considered for this problem are the Hölder spaces $C^{k, \alpha}(D)$, where $k$ is a positive integer, $0<\alpha \leqslant 1$, and $D$ is a domain in $\mathbf{R}^{3}$.

Let the boundary data $h$ belong to class $C^{0, \alpha}(S)$, for some $\alpha \in(0,1]$.

(a) Let $D=S \cup S_{\mathrm{e}}$. We will prove that $u \in C^{1, \alpha}(D)$, i.e., that

$$
\|u\|_{1, \alpha ; D} \leqslant \sum_{n=1}^{\infty} \varepsilon^{n-1}\left\|u_{n}\right\|_{1, \alpha ; D}<\infty, \quad\|\cdot\|_{1, \alpha ; D}=\|\cdot\|_{C^{1, \alpha}(D) .}
$$

For the exterior Neumann problem it is known [6, p. 313] from the Schauder estimates that, given

$$
\Delta w=0 \quad \text { in } S_{\mathrm{e}}, \quad(\partial w / \partial n)_{S}=\varphi,
$$

with $\varphi \in C^{0, \alpha}(S)$ for some $0<\alpha \leqslant 1$, then

$$
\|w\|_{1, \alpha ; S_{\mathrm{e}}} \leqslant K\|\varphi\|_{0, \alpha ; S},
$$

for some $K>0$. Moreover, the first derivatives of $w$ belong to class $C^{0, \alpha}(D)$ and are continuously extendable on $S$ from $S_{\mathrm{e}}$.

Therefore, from (4) and the continuity of the norm we get

$$
\|w\|_{1, \alpha ; D} \leqslant K\|\varphi\|_{0, \alpha ; S} .
$$

It is also known that $w$ is continuously extendable at each point of the surface $S$, and, since $w \in C^{1, \alpha}\left(S_{\mathrm{e}}\right)$, this extension belongs to class $C^{1, \alpha}(D)$ [6, p. 77].

We can thus use the above results for all the boundary value problems given in (3). For the first equation we have

$$
\left\|u_{1}\right\|_{1, \alpha ; D} \leqslant \frac{K}{2}\|h\|_{0, \alpha ; S}
$$

with all derivatives of $u_{1}$, extended to $S$, of class $C^{0, \alpha}(D)$. This result and the recursive relation for the boundary conditions for the other $u_{n}$ 's imply

$$
\left(\nabla u_{k} \cdot \nabla u_{j}\right)_{S} \in C^{0, \alpha}(S), \forall k, j .
$$

From the definitions of the norms of the spaces $C^{0, \alpha}(S)$ and $C^{1, \alpha}(D)$ we prove that

$$
\left\|\nabla u_{k} \cdot \nabla u_{j}\right\|_{0, \alpha ; S} \leqslant 9\left\|u_{k}\right\|_{1, \alpha ; D}\left\|u_{j}\right\|_{1, \alpha ; D}, \quad \forall k, j .
$$

Therefore, combining (6) and (7) we finally obtain the following estimates:

$$
\begin{aligned}
& n=1:\left\|u_{1}\right\|_{1, \alpha ; D} \leqslant \frac{K}{2}\|h\|_{0, \alpha ; S}, \\
& n \geqslant 2:\left\|u_{n}\right\|_{1, \alpha ; D} \leqslant \frac{9 K}{2} \sum_{k=1}^{n-1}\left\|u_{k}\right\|_{1, \alpha ; D}\left\|u_{n-k}\right\|_{1, \alpha ; D}
\end{aligned}
$$

where $K$ is the constant given by (5).

Now we will construct a convergent series that majorizes the series

$$
\sum_{n=1}^{\infty} \varepsilon^{n-1}\left\|u_{n}\right\|_{1, \alpha ; D}
$$


This is analogous to the Cauchy-Kowalewsky idea of majorizing series by series that solve a simpler problem which is known to converge [3].

Looking at the boundary condition given in (II), it can be seen as a quadratic equation in the derivatives of $u, \varepsilon^{2} \nabla u \cdot \nabla u$ representing the second degree term, $2 \varepsilon \partial u / \partial n$ the first degree term, and $-\varepsilon h$ the constant term. This suggests constructing a majorizing series, which in turn is a solution of some quadratic equation with appropriate coefficients, in the same spirit as the Cauchy-Kowalewsky theorem.

Therefore, let us consider the quadratic equation

$$
A x^{2}+B x+\varepsilon C=0
$$

for some constants $A, B, C$ to be determined. Using the binomial expansion, we obtain a convergent series solution of the form

$$
x=\sum_{n=1}^{\infty} a_{n} \varepsilon^{n},
$$

provided

$$
4 A C \varepsilon<B^{2} .
$$

Substituting the series into the quadratic equation and equating to zero all the coefficients of equal powers of $\varepsilon$, we have

$$
\begin{aligned}
& n=1: \quad a_{1}=-\frac{C}{B} \\
& n \geqslant 2: \quad a_{n}=-\frac{A}{B} \sum_{k=1}^{n-1} a_{k} a_{n-k} .
\end{aligned}
$$

In order to have

$$
\left\|u_{n}\right\|_{1, \alpha: D} \leqslant a_{n}, \quad \forall n,
$$

we choose the constants $A, B, C$ of the following form:

$$
A=9 K, \quad B=-2, \quad C=K\|h\|_{0, \alpha: S,}
$$

where $K>0$ is the constant given in (5).

Convergence of the series is guaranteed provided condition (9) is fulfilled, that is, if

$$
9 \varepsilon K^{2}\|h\|_{0, \alpha ; S}<1 \text {. }
$$

Substituting (11) into (10) we can now compare the series for $u$ with the series for $x$ by using the estimates in (8).

Hence

$$
\begin{aligned}
n=1: \quad\left\|u_{1}\right\|_{1, \alpha ; D} & \leqslant \frac{K}{2}\|h\|_{0, \alpha ; S}=a_{1}, \\
n \geqslant 2: \quad\left\|u_{n}\right\|_{1, \alpha ; D} & \leqslant \frac{9 K}{2} \sum_{k=1}^{n-1}\left\|u_{k}\right\|_{1, \alpha ; D}\left\|u_{n-k}\right\|_{1, \alpha ; D} \\
& \leqslant \frac{9 K}{2} \sum_{k=1}^{n-1} a_{k} a_{n-k}=a_{n} .
\end{aligned}
$$


The last inequality is true since $u_{n}$ depends on $u_{1}$ for all $n, u_{1}$ is majorized by $a_{1}$, and the recursive relations defining $a_{n}$ and $u_{n}$ for $n \geqslant 2$ are the same and are positivity-preserving.

We conclude that

$$
\varepsilon\|u\|_{1, \alpha ; D} \leqslant \sum_{n=1}^{\infty} \varepsilon^{n}\left\|u_{n}\right\|_{1, \alpha ; D} \leqslant \sum_{n=1}^{\infty} \varepsilon^{n} a_{n}=x<\infty
$$

for the boundary data $h \in C^{0, \alpha}(S)$ and the small parameter $\varepsilon$ satisfying

$$
\varepsilon\|h\|_{0, \alpha: S}<\frac{1}{9 K^{2}} .
$$

Therefore

$$
u \in C^{1, \alpha}(D)
$$

(b) Relation (13) implies uniform convergence of the series $u$. All the terms $u_{n}$ of this series are harmonic functions in $S_{\mathrm{e}}$ and continuous on $S$; therefore the limit $u$ of the series is also harmonic in $S_{\mathrm{e}}$ and continuous on $S$.

(c) The functions $u_{n}$ are solutions of exterior Neumann problems on the unit sphere. Therefore, they converge to zero at infinity. Since the convergence of the series $u$ is uniform we obtain that the series also converges to zero at infinity.

(d) Finally, the series $u$ satisfies the boundary condition (II) by construction.

4. Conclusions. In Sec. 2 we reformulated the original nonlinear problem (I) into the nonlinear problem (II) by perturbing the gravitational potential as a dominant known field plus a small unknown field.

The dominant known field generated a sequence of linear problems with known solutions. These solutions were combined as a power series in the small parameter $\varepsilon$ used in the perturbation.

Assuming the boundary data $h$ of class $C^{0, \alpha}(S)$ we proved local convergence of the series $u$ provided

$$
\varepsilon\|h\|_{0, \alpha ; S}<\frac{1}{9 K^{2}}
$$

Nevertheless, outside this region of convergence the behavior of the series is unknown and more research needs to be done in this direction.

We chose to prove existence of the solution by means of a power series instead of using the Implicit Function Theorem, because of its constructive approach. In this way, a numerical implementation to approximate the constructed solution is straightforward and does not require any numerical sophistications. 
Acknowledgments. The author wishes to thank A. A. Minzoni for his valuable suggestions and careful revision of this work. During this research the author was supported by a CONACYT Scholarship.

\section{REFERENCES}

[1] G. E. Backus, Application of a non-linear boundary value problem for Laplace's equation to gravity and geomagnetic intensity surveys, Quart. J. Mech. Appl. Math. 21, 195-221 (1968)

[2] G. E. Backus, Non-uniqueness of the external geomagnetic field determined by surface intensity measurements, J. Geophys. Res. 75, 6339-6341 (1970)

[3] R. Courant and D. Hilbert, Methods of mathematical physics, Vol. 2., Interscience, New York, 49, 1962

[4] N. M. Günter, Potential theory, Dover, New York, 1967

[5] O. D. Kellogg, Foundations of potential theory, Dover, New York, 1953

[6] V. D. Kupradze, Three dimensional problems of the mathematical theory of elasticity and thermoelasticity, North-Holland Publishing Company, Amsterdam, 1976 